\title{
A UME Kibble balance displacement measurement procedure
}

\author{
Haci Ahmedov' ${ }^{1}$, Mehmet Çelik ${ }^{1}$, Recep Orhan ${ }^{1}$, Beste Korutlu ${ }^{1}$, Ramiz Hamid $^{1}$, Ersoy Şahin ${ }^{1}$ \\ ${ }^{1}$ TÜBITAK National Metrology Institute (UME), Kocaeli, TURKEY
}

\begin{abstract}
The redefinition of the kilogram in terms of Planck constant came into effect on 20 May 2019. The National Metrology Institute of Turkey (UME) realised the new definition by means of the oscillating magnet Kibble balance. The novel dynamical measurement procedure developed for Kibble balance in Turkey has the advantage of being less sensitive to environmental disturbances compared to the traditional Kibble balance experiments. Precise displacement measurements are performed either with Michelson or Fabry-Perot interferometers in worldwide Kibble balances. Moreover, most of them operate in a global vacuum. A commercial Michelson interferometer has been used in UME's Kibble balance experiment. In this article, we determine the contribution of ultra-small oscillations to the Planck constant by taking simultaneous displacement measurements on two back-to-back mirrors attached to the piezoelectric transducer, undergoing an oscillatory motion with the Michelson and Fabry-Perot interferometers. The following novel measurement procedure makes such measurements possible in a regular laboratory environment. Otherwise, the experiment needs to be performed in a global vacuum. This is why we were required to investigate the resolution performances of these devices in laboratory conditions. As the expected relative uncertainty in the redefinition of kilogram is above the resolution uncertainties of both interferometers, we may conclude that a commercial Michelson interferometer will serve our purposes in our route to the redefinition of a kilogram by means of local vacuum.
\end{abstract}

\section{Section: RESEARCH PAPER}

Keywords: Kibble balance; Planck constant; interferometer; SI unit; resolution uncertainty

Citation: Haci Ahmedov, Mehmet Çelik, Recep Orhan, Beste Korutlu, Ramiz Hamid, Ersoy Sahin, A UME Kibble Balance Displacement Measurement Procedure, Acta IMEKO, vol. 9, no. 3, article 3, September 2020, identifier: IMEKO-ACTA-09 (2020)-03-03

Editor: Jan Saliga, Technical University of Košice, Slovakia

Received January 9, 2020; In final form April 1, 2020; Published September 2020

Copyright: This is an open-access article distributed under the terms of the Creative Commons Attribution 3.0 License, which permits unrestricted use, distribution, and reproduction in any medium, provided the original author and source are credited.

Funding: This work was supported by TÜBiTAK National Metrology Institute, Turkey.

Corresponding author: Haci Ahmedov, e-mail: haji.ahmadov@tubitak.gov.tr

\section{INTRODUCTION}

On 16 November 2018, a crucial decision on the redefinition of the kilogram unit was made according to the votes of the member states of the International Bureau of Weights and Measures (BIPM) - at the $26^{\text {th }}$ meeting of The General Conference on Weights and Measures (CGPM) held in Versailles, France. Based on the affirmative vote, effective from 20 May 2019, the Planck constant with a fixed value of $h=$ $6.62607015 \times 10^{-34} \mathrm{~J} \mathrm{~s}$ is used in the new definition of the kilogram, thereby ensuring a uniform, globally accessible, longterm stable unit for mass measurements rather than a physical artefact with limited access and quantitative limitations - namely the International Prototype of the Kilogram (IPK). The new definition of the kilogram can be realised by means of the Kibble balance experiment [1], which compares electrical power to mechanical power with the help of two macroscopic electrical quantum phenomena: the Quantum Hall effect [2] and the
Josephson effect [3]. Different construction and operating modes in Kibble balance experiments (see [4] and the citations therein) are favourable for gaining a thorough understanding of possible systematic errors. The TÜBİTAK National Metrology Institute (UME) of Turkey contributes to the ongoing worldwide scientific work on the redefinition of the kilogram, with an oscillating magnet Kibble balance experiment, in which its novel dynamical measurement procedure makes the system less sensitive to environmental disturbances compared to the traditional Kibble balance experiments [5]-[7].

All existing Kibble balances consist of a coil suspended from a balance embedded in a strong magnetic field created by a permanent magnetic circuit. The relative motion between the coil and the magnetic circuit induces Faraday's voltage across the ends of the coil. The precise measurements of the displacement and the voltage are vital in order to achieve the required total relative uncertainty of $2 \times 10^{-8}$ in the kilogram realization experiments with Kibble balance systems. This article focuses on the displacement measurements. In the Kibble balance 
experiments, laser interferometers, e.g. the Michelson interferometer (MI) [8]-[14] and the Fabry-Perot interferometer (FPI) [15] are used to measure the displacement between the magnetic circuit and the coil (see Table 2 in [4] and the citations therein). In the MI, the laser beam is split into two perpendicular arms, the reference arm and the measurement arm, and recombines at the beam splitter after being reflected by the reference and measurement reflectors, respectively. The phase differences between the two beams are influenced by tiny variations in environmental changes, as they follow different optical paths. The FPI, on the other hand, includes only one arm with two parallel mirrors aligned to form an optical resonator. As it features a common optical path, the FPI is less sensitive to environmental disturbances than the MI [16]. The factors determining the accuracy, linearity, and repeatability of highresolution displacement measurements are thoroughly investigated in [17]. In traditional Kibble balances, extreme precautions are taken for eliminating the environmental disturbances in the displacement measurements between the moving coil and the stationary magnet such that the entire system (all mechanical components, including the weighing cell; the balance pan and connecting components, coils, and magnet; and the interferometer) is covered by a global vacuum of less than 0.1 $\mathrm{Pa}$. Hence, the stability of the refractive index along the paths of the laser beams is ensured. In moving-coil Kibble balance experiments, a global vacuum is the only possibility for precise displacement measurements with interferometer optics. One disadvantage of this design is that the vacuum complicates the experiment. Firstly, all components in the experimental setup should be compatible with the vacuum. The second challenge is the dissemination of a mass unit. Since the mass unit is realised in a vacuum but disseminated in the air, one should consider the sorption effects on the reference mass calibrated in the Kibble balance experiment with a vacuum enclosure before using it for the dissemination of the kilogram [18]-[23]. A more direct method for disseminating the mass unit realised in a vacuum has been developed at NIST, where the magnetic suspension mass comparator (MSMC) is used to perform direct comparison of a mass artefact in a vacuum to the one in the air [24]. However, one needs a transportable vacuum component to transfer the mass artefact from the NIST Kibble balance to the MSCS.

In this article, we apply the dynamical measurement procedure developed for the UME oscillating magnet Kibble balance experiment on interferometric displacement measurements performed by the MI and FPI simultaneously. We are mainly concerned with the measurement capabilities of the MI for ultra-small displacements, where environmental disturbances are common. The FPI is used to justify the ultrasmall displacement measurements taken by the MI. The results indicate that the measurement precision of the MI is immune to environmental disturbances thereon provided that the aforementioned measurement procedure is followed. This allows us to use a commercial miniature plane mirror MI with a compact sensor head for the precision displacement measurements in the UME oscillating magnet Kibble balance. Moreover, in the UME Kibble balance experiment, as the moving piece is the magnetic circuit rather than the coil, one may introduce a local vacuum (covering only the mirror on the magnet together with its tilt mechanism) on the mechanically decoupled magnetic circuit. Currently, a local vacuum is under construction for ensuring stability of the refractive index. It is important to note that it is the stationary coil setup of the UME Kibble balance and the short duration in the measurement procedure that allow the use of a local vacuum instead of a global one. In Section II, we introduce the novel measurement procedure developed for the UME Kibble balance experiment. In Section III, we present the results obtained with the MI and FPI simultaneously. Finally, in the concluding section, the major advantages of the UME Kibble balance are summarised.

\section{MEASUREMENT PROCEDURE}

The UME oscillating magnet Kibble balance consists of a stationary coil suspended from the handler of the mass comparator and a mass standard of $1 \mathrm{~kg}$ on the handler. The coil is placed in the air gap of an oscillating magnetic circuit along the direction of the gravitational acceleration. Ampere's force law and Faraday's law of induction play central roles in this configuration such that the gravitational force on the mass standard is counter balanced by the Lorentz force $F$, created on the coil carrying a DC current $J$, through radially outward magnetic flux density. The oscillating magnetic circuit with the velocity $u(t)$ induces an AC Faraday's voltage $V(t)$ across the ends of the coil. Combining these two laws and assuming the velocity $u(t)$ is directed along the direction of gravitational acceleration (no horizontal or angular motions), one may write

$$
\frac{h}{h_{\mathrm{c}}} V(t)=G u(t)
$$

where $h$ is the actual Planck constant, $h_{\mathrm{c}}$ is the consensus value of the Planck constant, as announced in the $26^{\text {th }}$ CGPM meeting. The ratio $h / h_{\mathrm{c}}$ on the left hand side of Equation (1) appears due to the fact that the voltage and resistance are measured based on the Josephson and Klitzing constants, which are determined by using the consensus value of the Planck constant. $G=F / J$ is the geometrical factor, which depends on the structure of the magnetic flux density and the geometry of the coil.

In the context of this study, we may neglect the inhomogeneity of the geometrical factor in the vertical direction. Following this assumption, the measurement procedure followed in the UME Kibble balance yields

$$
\frac{h}{h_{\mathrm{c}}}=G\{z \mid W\},
$$

where $z=\int u d t$ is the displacement of the magnet, $W=$ $\int V d t$ is the magnetic flux passing through the coil, and

$$
\{z \mid W\}=\frac{1}{2 N} \sum_{k=1}^{2 N} \frac{z_{k+1}-z_{k}}{W_{k+1-} W_{k}},
$$

represents the averaging of the displacement over the magnetic flux (see [6] for details). A multiple of the fundamental period of oscillation has been chosen as the integration time of the Kibble balance experiment. We divide the integration time into $2 N$ halfcycles, denoted by $k$. One half-cycle is the region between the consecutive displacement extrema. For the $k^{\text {th }}$ half-cycle, $z_{k}$ is the preceding extrema, while $z_{k+1}$ is the succeeding one, as shown in Figure 1.

The corresponding $W_{k}$ and $W_{k+1}$ values are taken at the displacement extremum points. Using Equations (1) and (2), we obtain

$$
\frac{h^{(m)}}{h}=\left\{z^{(m)} \mid z\right\}
$$




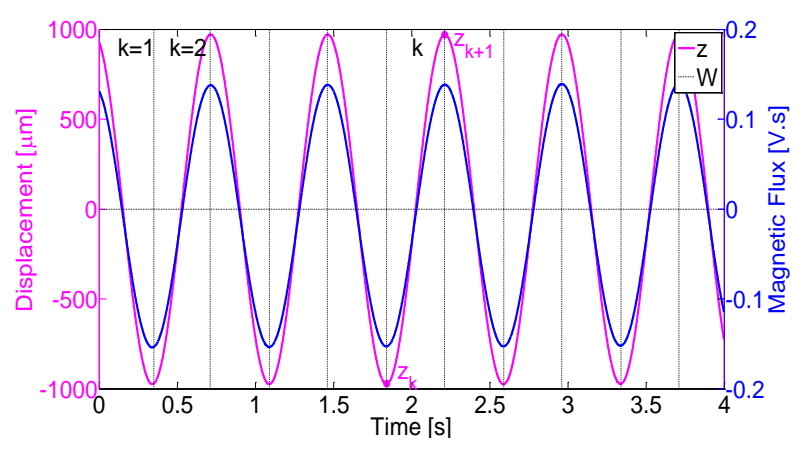

Figure 1. Synchronised data of the displacement and magnetic flux. A small portion of the data is presented for better illustration of the half-cycles.

where the parameters with the superscript $(m)$ are the measured values, and those without the superscript are the actual values of the corresponding parameters. The ratio of the measured Planck constant to the actual one is equal to the averaging of the measured displacement over the actual displacement. An ideal displacement measurement device would make this ratio equal to one. Any deviations from this value represent the inaccuracies of the measurement device. As we are interested in determining the displacement measurement uncertainties, in deriving Equation (4) we assume that the measurement uncertainties on all other contributing parameters (i.e. electrical current, voltage, mass, and gravity) are sufficiently small for the realisation of the kilogram.

The resolution of a measurement instrument is an essential property as it describes the smallest measurable quantity. In this article, we investigate the reading performance of the commercial MI used in the UME Kibble balance experiment. We use a sinusoidal reference signal with an ultra-small amplitude. As a reference signal $Z$, we use the oscillation produced by the piezoelectric transducer (PZT). The high stability of PZT allows us to describe the reference signal by only three parameters: fundamental oscillation frequency $f$, oscillation amplitude $A$, and phase $\varphi$, such that

$$
z(t)=A \cos (2 \pi f t+\varphi) .
$$

The fundamental oscillation frequency $f$ and the phase $\varphi$ are obtained by the signal processing followed in the next section. The oscillation amplitude $A$ is chosen to be close to the resolution of the MI. Since we neglect uncertainty over the full scale, we do not need to measure the amplitude $A$ of the ultrasmall oscillations exactly. We may rescale it to a desired value. In our analysis, we rescale the oscillation amplitude of PZT to be equal to that of the magnetic circuit in the UME Kibble balance experiment. In this case, Equation (4) defines the contribution of

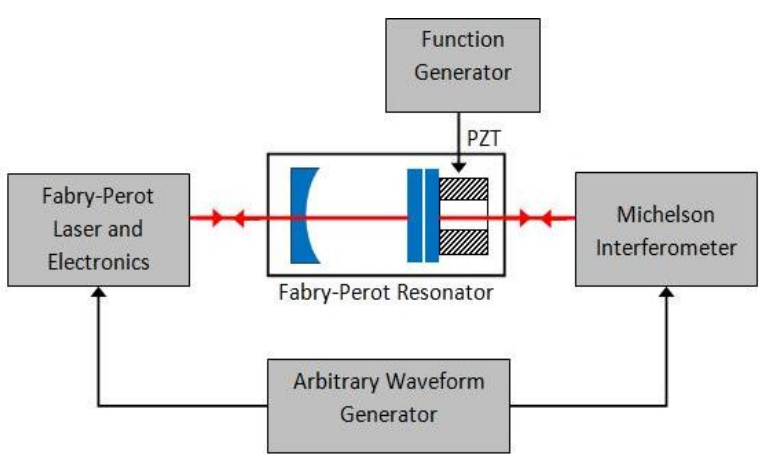

Figure 2. A schematic diagram of the experimental setup. ultra-small amplitude oscillations to the Planck constant, and its uncertainty defines the resolution uncertainty. In the rest of the article, we refer to the contribution of ultra-small amplitude oscillations to the Planck constant simply as 'ultra-small oscillation contributions'.

\section{RESULTS AND DISCUSSIONS}

The experimental setup consists of a SIOS AE SP 2000E miniature plane mirror MI and a UME-made FPI [25], [26], as shown in Figure 2. Two back-to-back mirrors attached to the PZT operate in an oscillatory motion. The frequency and amplitude of this motion is supplied by the AC voltage applied on the PZT by the function generator. The reading performance of $\mathrm{MI}$ is tested by using a reference signal with an amplitude of about $10 \mathrm{pm}$. This value is given in the specifications of the device as the resolution. In order to achieve such ultra-small amplitudes, in-line attenuators are used at the output of the function generator. The oscillations of about $1 \mathrm{~Hz}$ are measured by FPI and MI simultaneously for a time interval of $500 \mathrm{~s}$. The oscillation frequency is chosen to be equal to the $1 \mathrm{~Hz}$ oscillation frequency of magnetic circuit in UME Kibble balance experiment [5]-[7]. A Keysight 33512B Two-Channel Arbitrary Waveform Generator is used for the synchronisation of the FPI and MI data samplings of $50 \mathrm{~Hz}$.

The displacement data for MI $\left(z_{n}^{\mathrm{M}}\right)$ and FPI $\left(z_{n}^{\mathrm{FP}}\right)$ are shown in Figure 3.

The signal discretization index $n$ runs from 1 to $N=25000$. In order to estimate the fundamental oscillation frequency $f$ and the phase $\varphi$ of the reference signal encoded in these curves, it is

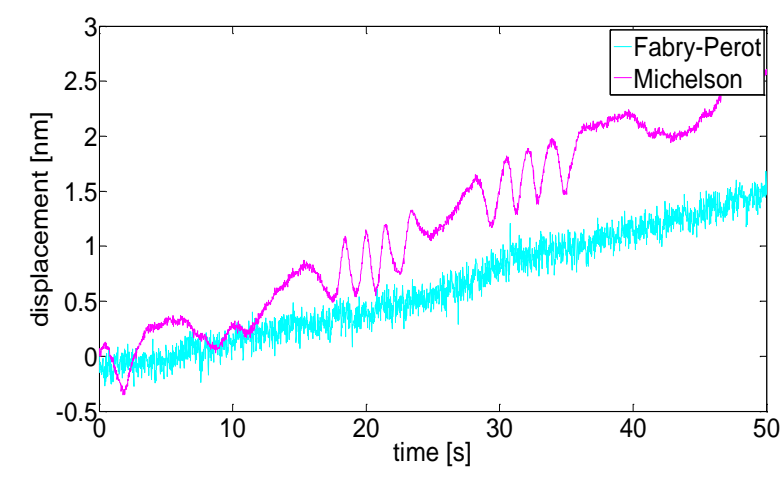

Figure 3. Displacement data measured by FPI (cyan) and MI (magenta). For illustration purposes, only a portion of the data is shown.

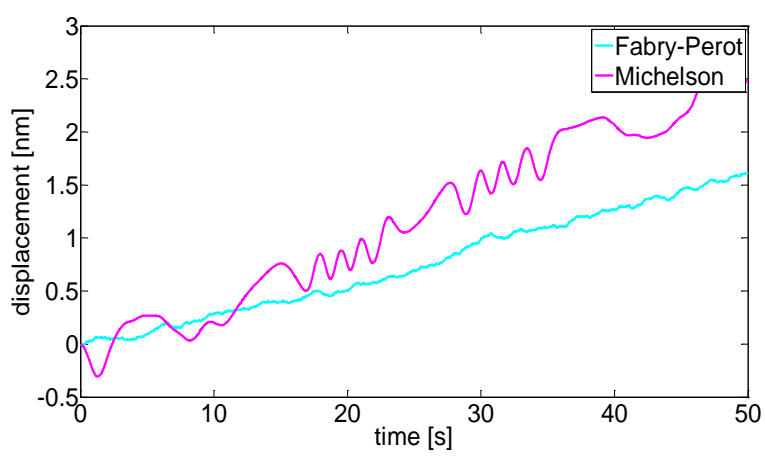

Figure 4. The low-pass filtered data of FPI (cyan) and MI (magenta). For illustration purposes, only a portion of the data is shown. 
useful to decompose the data into two parts as digitally bandpass filtered data and its residual. Digitally band-pass filtered data $\tilde{z}_{n}^{\mathrm{M}(\mathrm{FP})}$ is of the form

$$
\tilde{z}_{n}^{\mathrm{M}(\mathrm{FP})}=\frac{1}{2 l+1} \sum_{s=-l}^{l} z_{n+s}^{\mathrm{M}(\mathrm{FP})},
$$

where $2 l+1$ is obtained by taking the ratio of the oscillation frequency to the sampling frequency and $n=l+1, \cdots, N-l$. The digitally band-pass filtered data given in Figure 4 describes the low-frequency processes that are caused mainly by environmental disturbances.

The four parameter sine-fit (FPSF) algorithm [27] is performed in order to determine the amplitude, frequency, and phase of the residual data measured by MI and FPI. The results are summarised in Table 1.

The residual FPI and MI data and the signal reconstructed by the FPSF algorithm are given in Figure 5 and Figure 6, respectively.

The findings of the analysis indicate that the parameters estimated by the FPSF algorithm on the MI and FPI residual data are quite close to each other (Table 1). This is a strong indication of the consistency between the interferometric measurements taken by MI and FPI. The differences occur due to the low signal-to-noise ratio of about $-20 \mathrm{~dB}$ in the residual data. It is reasonable to assume that the actual phase and frequency values are in close proximity to the obtained ones for the reference signal.

To establish the resolution performance of the interferometers for ultra-small oscillations, we divide the raw data $z^{\mathrm{MI}(\mathrm{FPI})}$ of $500 \mathrm{~s}$ into five independent measurement sets of $100 \mathrm{~s}$ and represent each set of displacement data by $z_{S}^{\mathrm{MI}(\mathrm{FPI})}$ where $s=1, \cdots, 5$. The ratio $h_{s}^{\mathrm{MI}(\mathrm{FPI})} / h$ is determined for each set using Equation (4). The mean value of these sets defines the contribution of the ultra-small amplitude oscillations to the Planck constant, and the standard deviation defines the resolution uncertainty.

As summarised in Table 1, the interferometric measurements of the ultra-small oscillations by MI and FPI are close to each other. Therefore, it is reasonable to expect ratio $h_{s}^{\mathrm{MI}} / h$ to be close to ratio $h_{s}^{\mathrm{FPI}} / h$. Consequently, one may perform the following optimisation procedure for estimating the parameters of the reference signal. As a first step, an optimisation function

$$
F(f, \varphi)=\sum_{s=1}^{5}\left(\frac{h_{s}^{\mathrm{FPI}}}{h}-\frac{h_{s}^{\mathrm{MI}}}{h}\right)^{2}
$$

Table 1. The frequency, amplitude, and phase obtained by the FPSF algorithm applied on the FPI and MI residual data.

\begin{tabular}{lccc}
\hline Parameters & FPI & MI & Difference \\
\hline Frequency $(\mathrm{Hz})$ & 1.00036 & 0.99987 & 0.00049 \\
Amplitude $(\mathrm{pm})$ & 8.5 & 8.1 & 0.4 \\
Phase $(\mathrm{rad})$ & 1.17 & 1.10 & 0.07 \\
\hline
\end{tabular}

Table 2. The mean values for the ultra-small oscillation contributions and resolution uncertainties for $\mathrm{FPI}$ and $\mathrm{MI}$.

\begin{tabular}{lcc}
\hline Parameters & FPI & MI \\
\hline Mean & $6.9 \times 10^{-9}$ & $7.2 \times 10^{-9}$ \\
Standard Deviation & $2.2 \times 10^{-9}$ & $1.4 \times 10^{-9}$ \\
\hline
\end{tabular}

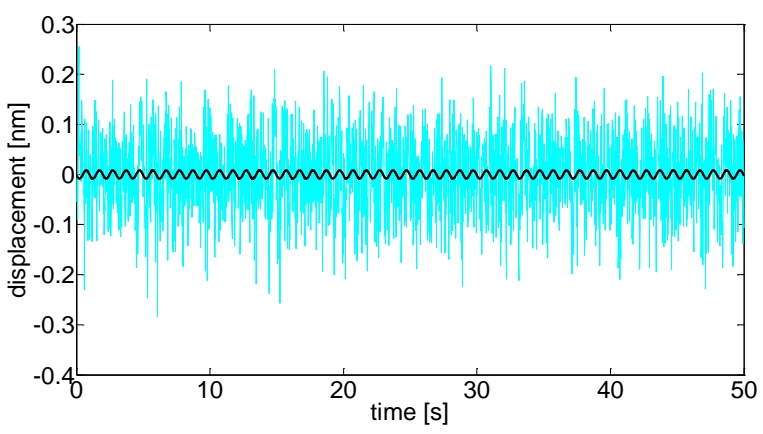

Figure 5. The residual data (cyan) measured by FPI and the estimation of the signal reconstructed by the FPSF algorithm performed on the residual data of FPI (black). For illustration purposes, only a portion of the data is given.

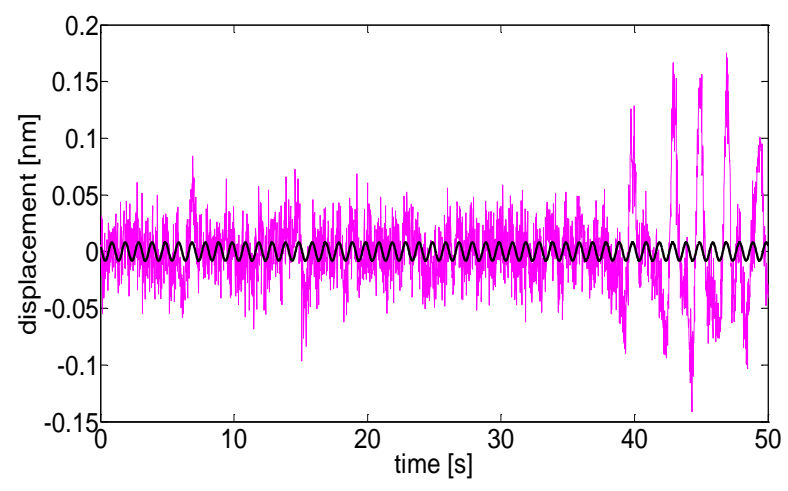

Figure 6. The residual data (magenta) measured by $\mathrm{MI}$ and the estimation of the reconstructed signal by FPSF algorithm performed on the residual data of $\mathrm{MI}$ (black). For illustration purposes, only a portion of the data is given.

is introduced. Then, using the optimisation function, $F(f, \varphi)$, a $100 \times 100$ matrix is constructed in light of the reconstructed signals, by increments of $0.00001 \mathrm{~Hz}$ for the frequency range from $0.99950 \mathrm{~Hz}$ to $1.00050 \mathrm{~Hz}$ and by increments of 0.01 rad for the phase range from $0.65 \mathrm{rad}$ to $1.65 \mathrm{rad}$. The estimated values for the reference signal are $f=1.00023 \mathrm{~Hz}$ and $\varphi=$ $0.95 \mathrm{rad}$ as they are the numerical values that make the minimum difference in Equation (7). Figure 7 summarises the ultra-small oscillation contributions obtained for each set $s$. The resolution uncertainties for the FPI and MI and the corresponding mean values determining the ultra-small oscillation contributions are summarised in Table 2. It is important to emphasise that even though the optimization procedure makes the results of MI and FPI agree better with each other, the mean value and the

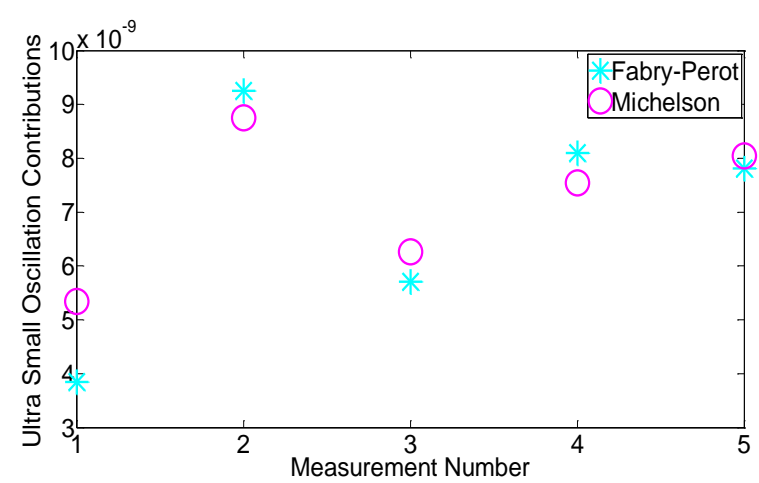

Figure 7. The contribution to $\mathrm{h}_{\mathrm{s}}^{\mathrm{MI}(\mathrm{FPI})} / \mathrm{h}$ obtained by simultaneous measurements with MI (circles in magenta) and FPI (stars in cyan). 
uncertainties obtained without the optimisation procedure are still below $10^{-8}$

In most of the existing Kibble balance experiments, the system operates within a global vacuum to ensure the stability of the index of refraction along the paths of the laser beams that measure the displacements of the moving coil with respect to the stationary magnet. The interlaced mechanical construction of these experiments restricts the vacuum to being global, as it is not possible to keep the coil itself within a separate vacuum. On the other hand, the UME oscillating magnet Kibble balance experiment can operate within a local vacuum. There are two fundamental reasons for this advantage. The first one is that in the UME Kibble balance experiment, the moving piece is the magnetic circuit - not the coil. As the magnetic circuit is not connected to the handler of the mass comparator, it could be treated as a separate piece. Hence, a local vacuum could be installed directly on the mechanically decoupled magnetic circuit. In Figure 8, the experimental setup with a local vacuum is shown, and the coil suspended from the handler of the mass comparator, the surrounding magnetic circuit, and the local vacuum placed on the magnet are indicated. For the purpose of better illustration, the middle section of the magnet where the coil resides is shown transparently. The second reason is that in displacement measurements, the uncertainty contribution due to the environmental conditions (fluctuation in the index of refraction and elongation of materials) are proportional to the measurement time. As the oscillation frequency of the magnetic circuit in the UME Kibble balance experiment might be increased, the time it takes for peak-to-peak displacements decreases. In such a short duration of time, the changes in the environmental conditions are sufficiently small, as it is shown in this article that a local vacuum stands as a practical application. Currently, the vacuum chamber to be placed on the magnetic circuit is under construction (Figure 9). It is fixed on the horizontal plate such that the vertical displacement of the magnet is measured in a medium with a constant index of refraction with a magnitude of one. The bellows between the surface of the

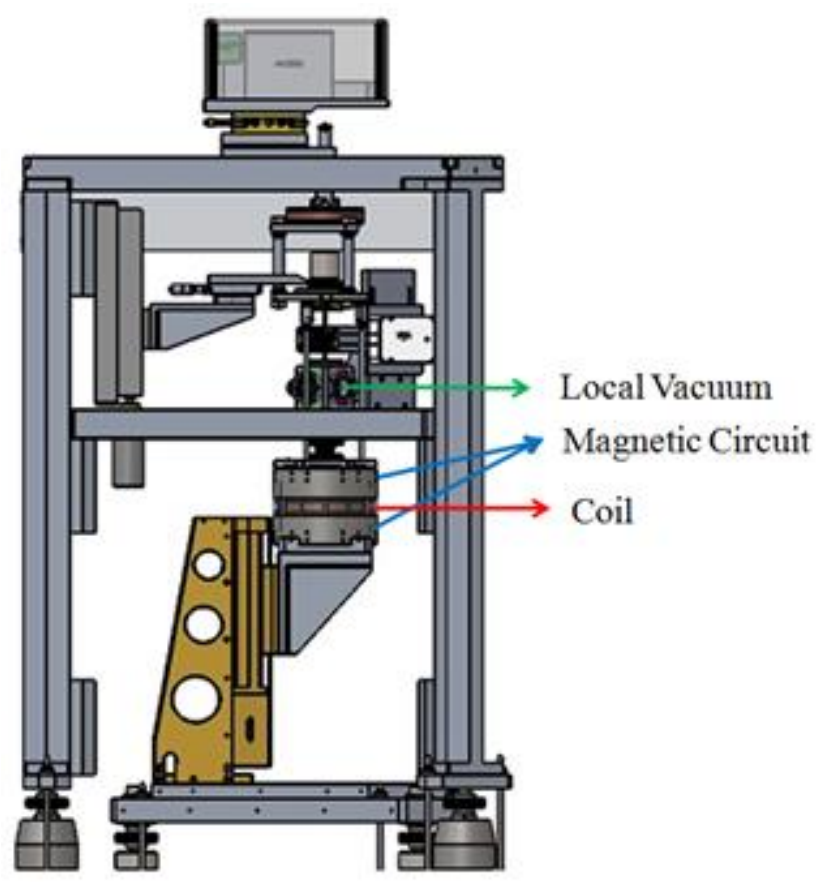

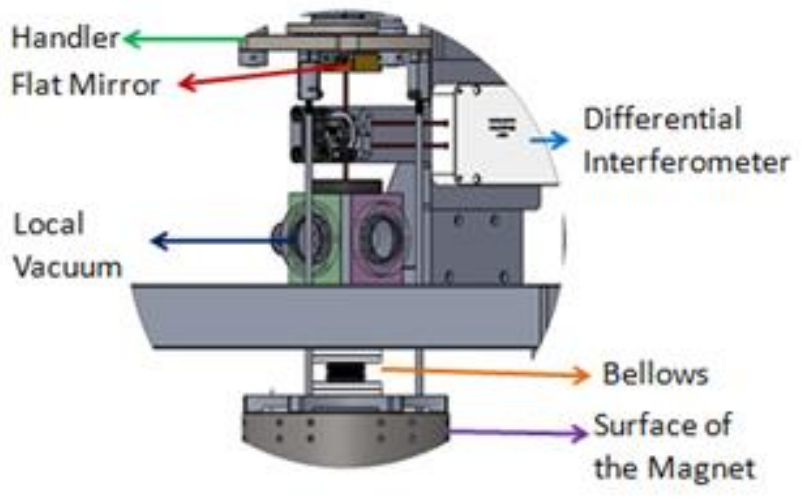

Figure 9. The local vacuum chamber in the UME Kibble balance experiment.

magnet and the bottom of the table are placed in order to introduce movement of the magnetic circuit into the vacuum.

In the final setup, we plan to use a SIOS differential interferometer for measuring the vertical displacements of the magnetic circuit whereby one of the beams is directed towards the flat mirror in the bottom of the handler, from which the coil is suspended and the second one is directed towards the upper surface of the cubic mirror within the local vacuum. The reason for using the cubic mirror is that the six degrees of freedom (three translations and three rotations) in the motion of the magnetic circuit could be traced via the cubic mirror. We plan to use a SIOS double-beam interferometer and a SIOS triple beam interferometer for measuring the horizontal and angular displacements of the magnetic circuit via the reflected beams of the two coincident side faces of the corner cube. In Figure 9, we show only the differential interferometer for the purpose of better illustration.

\section{CONCLUSIONS}

In this study, we applied the dynamical measurement procedure developed for the UME oscillating magnet Kibble balance to interferometric displacement measurements performed simultaneously by MI and FPI to test the resolution performance of MI. The data analysis yields mean values for the ultra-small oscillation contributions of $6.9 \times 10^{-9}$ and $7.2 \times$ $10^{-9}$, and resolution uncertainties of $2.2 \times 10^{-9}$ and $1.4 \times$ $10^{-9}$, for FPI and MI, respectively. As the relative uncertainty for the redefinition experiments of the kilogram is expected to be $2 \times 10^{-8}$, the ultra-small oscillation contribution and the resolution uncertainty obtained for the commercial miniature plane mirror MI will serve our purposes without the need to take extreme precautions for eliminating the environmental disturbances on the displacement measurements. The parasitic effects are eliminated simply by performing the dynamical measurement procedure developed for the UME Kibble balance experiment. This feature of measurement and the mechanically decoupled nature of the magnetic circuit allow us to use a local vacuum around the cubic mirror attached to the oscillating magnet as opposed to most of the existing Kibble balance experiments, in which a global vacuum is used. Currently, the local vacuum that we plan to use in the UME Kibble balance is under construction.

Figure 8. The UME Kibble balance experiment. 


\section{REFERENCES}

[1] B. P. Kibble, I. A. Robinson, Feasibility study for a moving coil apparatus to relate the electrical and mechanical SI units, Technical Report DES 40, NPL, 1977.

[2] K. von Klitzing, G. Dorda, M. Pepper, New method for high accuracy determination of the fine-structure constant based on quantised Hall resistance, Phys. Rev. Lett. 45 (1980) pp. 494-497. DOI: https://doi.org/10.1103/PhysRevLett.45.494

[3] B. D. Josephson, Possible new effects in superconductive tunnelling, Phys. Lett. 1 (1962) pp. 251-253.

DOI: https://doi.org/10.1016/0031-9163(62)91369-0

[4] I. A. Robinson, S. Schlamminger, The watt or Kibble balance: a technique for implementing the new SI definition of the unit of mass, Metrologia 53 (2016) pp. A46-74.

DOI: https://doi.org/10.1088/0026-1394/53/5/A46

[5] H. Ahmedov, An oscillating magnet watt balance, Proc. of the Conference on Precision Electromagnetic Measurements (CPEM 2016), Ottawa, Canada, 10-15 July 2016.

DOI: https://doi.org/10.1109/CPEM.2016.7540780

[6] H. Ahmedov, N. Babayiğit Aşkın, B. Korutlu, R. Orhan, Preliminary Planck constant measurements via UME oscillating magnet Kibble balance, Metrologia 55 (2018) pp. 326-333. DOI: https://doi.org/10.1088/1681-7575/aab23d

[7] H. Ahmedov, B. Korutlu, R. Orhan, Optimization procedure for Faraday's voltage in UME Kibble balance, IEEE Transactions on Instrumentation and Measurement 68(6) (2019) pp. 2172-2175. DOI: https://doi.org/10.1109/TIM.2019.2899185

[8] F. Bielsa, L. Robertsson, T. Lavergne, A. Kiss, H. Fang, M. Stock, The new interferometer of the BIPM watt balance, Proc. of the Conference on Precision Electromagnetic Measurements (CPEM 2016), Ottawa, Canada, 10-15 July 2016. DOI: https://doi.org/10.1109/CPEM.2016.7540576

[9] D. Kim, B. Woo, K. Lee, K. Choi, J. Kim, K. Wan, J. Kim, Design of the KRISS watt balance, Metrologia 51 (2014) S96 S100.

DOI: https://doi.org/10.1088/0026-1394/51/2/S96

[10] M. Thomas, D. Ziane, P. Pinot, R. Karcher, A. Imanaliev, F. Pereira Dos Santos, S. Merlet, F. Piquemal, P. Espel, A determination of the Planck constant using the LNE Kibble balance in air, Metrologia 54 (2017) pp. 468-480. DOI: https://doi.org/10.1088/1681-7575/aa7882

[11] C. M. Sutton, An oscillatory dynamic mode for a watt balance, Metrologia 46 (2009) pp. 467-472.

DOI: https://doi.org/10.1088/0026-1394/46/5/010

[12] I. A. Robinson, Towards the redefinition of the kilogram: a measurement of the Planck constant using the NPL Mark II watt balance, Metrologia 49 (2011) pp. 113-156. DOI: https://doi.org/10.1088/0026-1394/49/1/016

[13] B. M. Wood, C. A. Sanchez, R. G. Green, J. O. Liard, A summary of the Planck constant determinations using the NRC Kibble balance, Metrologia 54 (2017) pp. 399-409. DOI: https://doi.org/10.1088/1681-7575/aa70bf

[14] D. Haddad, F. Seifert, L. S. Chao, A. Possolo, D. B. Newell, J. R. Pratt, C. J. Williams, S. Schlamminger, Measurement of the Planck constant at the National Institute of Standards and Technology from 2015 to 2017, Metrologia 54 (2017) pp. 633-641.
Online [Accessed 24 September 2020]

DOI: https://doi.org/10.1088/1681-7575/aa7bf2

[15] A. Eichenberger, H. Baumann, B. Jeanneret, B. Jeckelmann, P. Richard, W. Beer, Determination of the Planck constant with the METAS watt balance, Metrologia 48 (2011) pp. 133-141. DOI: https://doi.org/10.1088/0026-1394/48/3/007

[16] W. Yung-Cheng, S. Lih-Horng, C. Chung-Ping, The comparison of environmental effects on Michelson and Fabry-Perot interferometers utilized for the displacement measurement, Sensors 10 (2010) pp. 2577-2586.

DOI: https://doi.org/10.3390/s100402577

[17] N. Bobroff, Recent advances in displacement measuring interferometry, Meas. Sci. Technol. 4 (1993) pp. 907-926. DOI: https://doi.org/10.1088/0957-0233/4/9/001

[18] P. Richard, H. Fang, R. Davis, Foundation for the redefinition of the kilogram, Metrologia 53 (2016) A6-A11. DOI: https://doi.org/10.1088/0026-1394/53/5/A6

[19] W. Beer, W. Fasel, E. Moll, P. Richard, U. Schneiter, R. Thalmann, J. Egger, The METAS $1 \mathrm{~kg}$ vacuum mass comparator-adsorption layer measurements on gold-coated copper buoyancy artefacts, Metrologia 39 (2002) pp. 263-268. DOI: https://doi.org/10.1088/0026-1394/39/3/3

[20] S. Davidson, Determination of the effect of transfer between vacuum and air on mass standards of platinum-iridium and stainless steel, Metrologia 47 (2010) pp. 487-497. DOI: https://doi.org/10.1088/0026-1394/47/4/015

[21] S. Davidson, J. Berry, P. Abbott, K. Marti, R. Green, A. Malengo, L. Nielsen, Air-vacuum transfer; establishing traceability to the new kilogram, Metrologia 53 (2016) pp. A95-A113. DOI: https://doi.org/10.1088/0026-1394/53/5/A95

[22] H. Wei, J. Pomeroy, Application of the double paddle oscillator for quantifying environmental, surface mass variation, Metrologia 53 (2016) pp. 869-880.

DOI: https://doi.org/10.1088/0026-1394/53/2/869

[23] A. Picard, H. Fang, Mass comparisons using air buoyancy artefacts, Metrologia 41 (2004) pp. 330-332. DOI: https://doi.org/10.1088/0026-1394/41/4/015

[24] Z. J. Jabbour, P. Abbott, E. Williams, R. Liu, V. Lee, Linking air and vacuum mass measurement by magnetic levitation, Metrologia 46 (2009) pp. 339-344.

DOI: https://doi.org/10.1088/0026-1394/46/3/023

[25] M. Celik, R. Hamid, U. Kuetgens, A. Yacoot, Picometre displacement measurements using a differential Fabry-Perot optical interferometer and an x-ray interferometer, Measurement Science and Technology 23(8) (2012) p. 085901.

DOI: https://doi.org/10.1088/0957-0233/23/8/085901

[26] M. Pisani, A. Yacoot, P. Balling, N. Bancone, C. Birlikseven, M. Çelik, J. Flügge, R. Hamid, P. Köchert, P. Kren, U. Kuetgens, A. Lassila, G. B. Picotto, E. Şahin, J. Seppä, M. Tedaldi, Chr. Weichert, Comparison of the performance of the next generation of optical interferometers, Metrologia 49(4) (2012) pp. 455-467.

DOI: https://doi.org/10.1088/0026-1394/49/4/455

[27] P. Händel, Properties of the IEEE-STD-1057 four-parameter sine wave fit algorithm, IEEE Transactions on Instrumentation and Measurement 49(6) (2000) pp. 1189-1193.

DOI: https://doi.org/10.1109/19.893254 